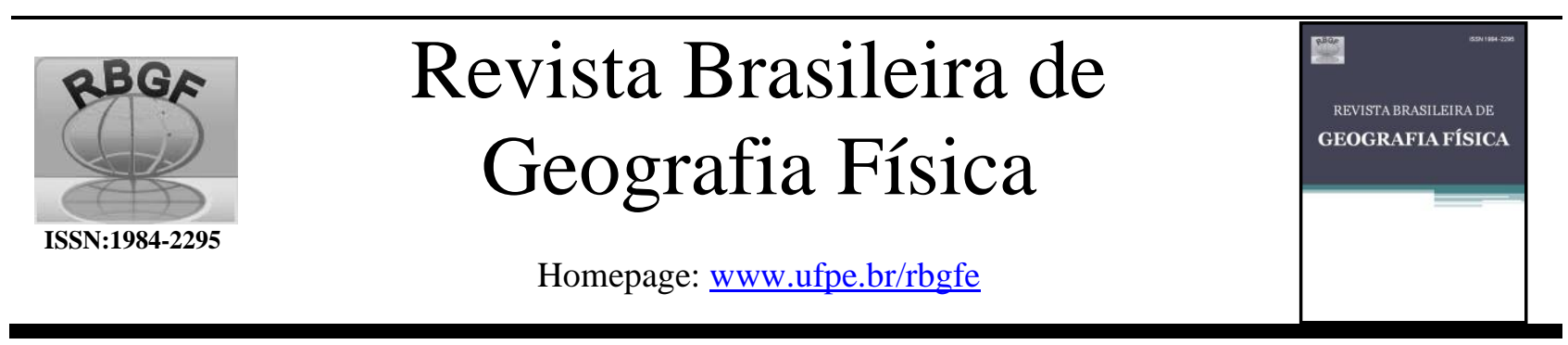

\title{
Relação entre o Vapor D’Água Atmosférico e a Temperatura da Superfície do Mar Sobre a Região da Confluência Brasil-Malvinas, com Base em Dados Coletados In Situ
}

\author{
Rose Ane Pereira de Freitas ${ }^{1}$; Ronald Buss de Souza $^{2}$ e Rafael Afonso do Nascimento Reis ${ }^{3}$
}

${ }^{1}$ Pós-doutoranda, Centro Regional Sul - Instituto Nacional de Pesquisas Espaciais (CRS-INPE); Av. Roraima s/n, Campus da UFSM, prédio do INPE, Bairro Camobi, Santa Maria, RS, Brasil, CEP 97105-900, rosefreitas78@gmail.com (autor correspondente). ${ }^{2}$ Doutor em Oceanografia física e Pesquisador Titular III, Centro Regional Sul - Instituto Nacional de Pesquisas Espaciais (CRS-INPE); Av. Roraima s/n, Campus da UFSM, prédio do INPE, Bairro Camobi, Santa Maria, RS, Brasil, CEP 97105-900, ronald.buss@inpe.br. ${ }^{3}$ Doutorando em Meteorologia Aplicada, Universidade Federal de Viçosa, Departamento de Engenharia Agrícola, P. H. Holfs S/N, 36570-000, Viçosa, MG, Brasil, cgb1504@gmail.com.

Artigo recebido em 21/03/2019 e aceito em 22/09/2019

\section{RES U M O}

O objetivo deste trabalho foi analisar a influência dos gradientes de TSM e da passagem de sistemas atmosféricos transientes sobre a região da Confluência Brasil-Malvinas (CBM) no Oceano Atlântico Sudoeste e descrever a variabilidade espacial e temporal da concentração de vapor d'água dentro da camada limite atmosférica marinha (CLAM). Os dados foram obtidos a partir de 130 perfis de radiossondas realizados durante dez cruzeiros oceanográficos feitos durante os meses de outubro e novembro entre 2004 e 2015. Os resultados mostram que os gradientes termais entre as águas quentes da Corrente do Brasil e as águas frias da Corrente das Malvinas foram capazes de produzir diferenças significativas no conteúdo de vapor d'água na CLAM nos dois lados da frente oceanográfica. Na superfície, o valor médio da umidade específica sobre o lado quente (frio) foi 8,4 $\pm 1,67 \mathrm{~mm}(7,08 \pm 1,51 \mathrm{~mm})$. Em todas as observações realizadas, a umidade integrada na CLAM foi diretamente influenciada pela advecção e pela passagem de sistemas atmosféricos transientes.

Palavras-chave: Vapor d’água; Confluência Brasil-Malvinas; Camada Limite Atmosférica

\section{Relationship between Atmospheric Water Vapor Content and the Sea Surface Temperature in the Brazil-Malvinas Confluence considering Data Collected In Situ}

\begin{abstract}
A B S T R A C T
The objective of this work was to analyze the influence of the SST gradients and the passage of transient atmospheric systems at the Brazil-Malvinas Confluence (BMC) region in the Southwest Atlantic Ocean, and to describe the spatial and temporal variability of the water vapor concentration within the marine atmospheric boundary layer (MABL). The data were obtained from 130 radiosondes profiles taken during ten oceanographic cruises carried out during the months of October and November between 2004 and 2015. The results show that the thermal gradients between the warm waters of Brazil Current and the cold waters of the Malvinas Current were able to produce significant differences in the water vapor content of the MABL on both sides of the oceanographic front. On the surface, the average of the specific humidity over the warm (cold) side was $8.4 \pm 1.67 \mathrm{~mm}(7.08 \pm 1.51 \mathrm{~mm})$. In all the observations made, the water vapor integrated in the MABL was directly influenced by the advection and by the passage of transient atmospheric systems.

Key words: Water Vapor; Brazil-Malvinas Confluence; Atmospheric boundary layer
\end{abstract}

Freitas; R. A. P., Souza, R. B., Reis, R. A. N. 


\section{Introdução}

O vapor d'água desempenha papel fundamental nos processos físicos que ocorrem na atmosfera, por exemplo, nos processos de transferência de radiação na atmosfera e no transporte e liberação de calor latente. Para o sistema climático global, o vapor d'água é essencial por afetar diretamente o balanço de energia da superfície terrestre por meio dos fluxos radiativos e da formação de nuvens, além de estabelecer uma ligação entre a superfície do planeta e a atmosfera. Essa ligação é feita através de um ciclo hidrológico que envolve o importante processo de realimentação, pois, à medida que aumenta a temperatura da atmosfera, mais vapor d'água pode ser armazenado (Nygârd et al., 2013).

Pezzi et al. (2005) mostraram que, na ausência dos sistemas de grande escala atuantes na região da CBM, a CLA será modulada pela frente oceânica que caracteriza a região. No lado norte da $\mathrm{CBM}$, as águas mais quentes induzem mudanças na estabilidade estática da CLA, que afetam a variabilidade na magnitude e intensidade dos ventos, enquanto o cisalhamento vertical do vento na CLA é reduzido, consequentemente ventos mais intensos ocorrem na superfície do mar, e a CLA se torna turbulenta e instável sobre águas mais quentes, aumentando a transferência de momentum para baixo.

Pezzi et al. (2016), e Camargo et al. (2013) mostraram a importância da região da CBM para a modulação de processos atmosféricos como fluxos de calor, estabilidade da camada limite atmosférica e a magnitude dos ventos, por exemplo. Os autores descrevem importantes processos e fenômenos ligados ao acoplamento oceano-atmosfera nessa região do Oceano Global, porém esses trabalhos não se interessaram em estudar com maior profundidade a concentração de vapor d'água na atmosfera e os efeitos dos gradientes de TSM locais e a atividade sinótica atmosférica sobre essa variável.

O Oceano Atlântico Sul (OAS), ainda que pouco estudado, é de fundamental importância no tempo e no clima do Brasil para a compreensão das variações dos fluxos de calor, umidade e momentum, e tem incentivado um crescente número de estudos sobre os processos de interação Oceano-Atmosfera no OAS, especialmente na região da sua parte ocidental, conhecida como Confluência Brasil Malvinas (CBM), mostrada a seguir na Figura 1, e sua consequente influência no tempo e no clima da América do Sul (Pezzi et al., 2016; Freitas et al, 2017).
Neste artigo são avaliados, com base em dados tomados in situ, dados de satélite e dados de reanálises, o papel dos gradientes de TSM e da passagem de sistemas atmosféricos transientes de escala sinótica sobre a região da CBM em determinar a variabilidade espacial e temporal da concentração de vapor d'água dentro da camada limite atmosférica marinha (CLAM) na região, gerando novas informações sobre o papel dos gradientes de TSM sobre o vapor d'água atmosférico sobre o Oceano Atlântico Sul.

\section{Material e métodos}

\section{Área de estudo}

Sendo o Oceano Atlântico Sul uma região de fundamental importância no tempo e clima do Brasil, ainda que pouco estudado, a área de interesse deste estudo se concentra na sua parte Sudoeste, conhecida como Confluência BrasilMalvinas (CBM).

A Figura 1 mostra a região de estudo e as estações de amostragem nos cruzeiros feitos por navios entre 2004 e 2015 para a coleta de dados. Esta região se caracteriza pelo encontro de águas mais frias da Corrente das Malvinas (CM) com águas mais quentes da Corrente do Brasil (CB), com alta variabilidade espacial e temporal, típica de regiões de frentes oceanográficas, sendo uma das mais energéticas frentes do ponto de vista oceanográfico. A região tem papel fundamental na modulação da CLAM, assim como nos fluxos de calor na interface oceano-atmosfera (Pezzi et al., 2016; Freitas et al., 2017).

\section{Dados In situ}

Os dados in situ usados neste estudo estão detalhados na Tabela 1 e foram amostrados no âmbito do INTERCONF (Estudo da Interação Oceano-Atmosfera na Região da Confluência Brasil-Malvinas). Este projeto tem por objetivo geral estudar a dinâmica da região oceânica e atmosférica do Oceano Atlântico Sudoeste e sua relação com os processos oceânicos e atmosféricos dos ambientes de altas latitudes no Oceano Atlântico e Austral. 


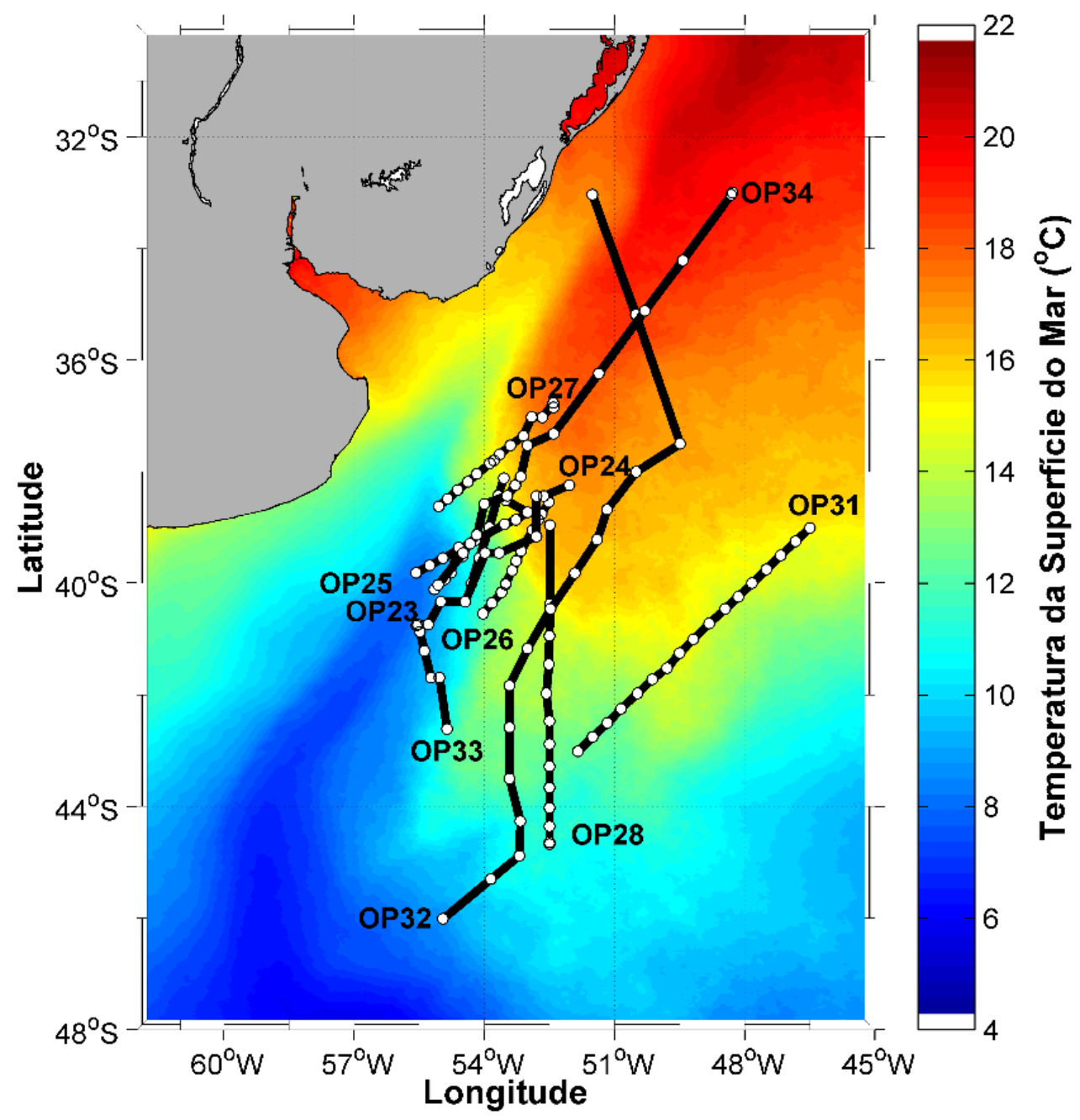

Figura 1 Região de estudo na CBM e localização das estações de amostragem in situ do Programa INTERCONF entre 2004 e 2015. A TSM da região é representada pela TSM média para o mês de outubro entre os anos de 2002 a 2014. Os respectivos acrônimos, variando de OP23 a OP34, representam os diferentes cruzeiros oceanográficos feitos para a coleta dos dados conforme Tabela 1. OP29 e OP30 não houve coleta de dados de radiossondas (Adaptado de Freitas et al, 2017).

Através do INTERCONF e do INCT da Criosfera, dados atmosféricos e oceanográficos têm sido coletados simultaneamente a cada Operação Antártica (OP), desde o ano de 2004 (OP23) até os dias de hoje, normalmente nos meses de outubro ou novembro de cada ano na região de estudo (Pezzi et al., 2016). O projeto atua como

\section{Dados Atmosféricos e Oceanográficos}

Durante as Operações Antárticas, da OP23 até OP31, os dados foram coletados in situ por sistemas de recepção de dados previamente instalados nos navios pelos pesquisadores durante parte das atividades desenvolvidas pelo PROANTAR a bordo de navios da Marinha do Brasil, como o navio de apoio oceanográfico NApOc Ary Rongel (H-44) e o navio de pesquisa polar Npo Almirante Maximiano (H-41) durante a realização das Operações Antárticas.

a realização dos cruzeiros, e o sistema de medição das condições atmosféricas usado foi o Vaisala DigiCORA II MW15. Tendo como referência a OP32 (2013), com a instalação de um sistema próprio no NPo. Almirante Maximiano (H-41), o sistema receptor utilizado passou a ser o Vaisala 
DigiCORA MW31. Os tipos de radiossondas usados operam na banda de frequência $400 \mathrm{MHz}$ e medem no mínimo as variáveis pressão, temperatura e umidade relativa do ar, direção e intensidade do vento.

Os dados oceanográficos foram coletados pelos seguintes instrumentos: para medidas de temperatura em função da profundidade, utilizouse o XBT (Expendable Bathy Thermograph); para medidas de condutividade, temperatura e pressão, utilizou-se o CTD (Conductivity Temperature Depth). Esta medida de condutividade pode ser corrigida com a temperatura para fornecer perfil de salinidade. E para a aquisição contínua de dados de temperatura e salinidade na superfície do mar, utilizou-se o Termossalinógrafo, que é instalado no casco do navio e permite a cada 10 segundos essa aferição ao longo da trajetória realizada pelo navio, cruzando a CBM.

\begin{tabular}{ccccc}
\hline & $\begin{array}{c}\text { Data de coleta } \\
\text { de dados }\end{array}$ & $\begin{array}{c}\text { Lat/Lon } \\
\text { Início do transecto }\end{array}$ & $\begin{array}{c}\text { Lat/Lon } \\
\text { Final do transecto }\end{array}$ & $\begin{array}{c}\text { Radiossondas (RS) } \\
\text { lançadas }\end{array}$ \\
\hline OP23 / 2004 & $02-03 / 10 / 2004$ & $38.12^{\circ} \mathrm{S} / 53.55^{\circ} \mathrm{W}$ & $40.01^{\circ} \mathrm{S} / 54.30^{\circ} \mathrm{W}$ & 5 \\
$\mathrm{OP} 24 / 2005$ & $28-29 / 10 / 2005$ & $38.54^{\circ} \mathrm{S} / 52.51^{\circ} \mathrm{W}$ & $40.54^{\circ} \mathrm{S} / 54.03^{\circ} \mathrm{W}$ & 12 \\
$\mathrm{OP} 25 / 2006$ & $27-28 / 10 / 2006$ & $38.51^{\circ} \mathrm{S} / 53.51^{\circ} \mathrm{W}$ & $39.81^{\circ} \mathrm{S} / 55.57^{\circ} \mathrm{W}$ & 10 \\
$\mathrm{OP} 26 / 2007$ & $16-17 / 10 / 2007$ & $39.52^{\circ} \mathrm{S} / 54.50^{\circ} \mathrm{W}$ & $40.11^{\circ} \mathrm{S} / 55.15^{\circ} \mathrm{W}$ & 5 \\
$\mathrm{OP} 27 / 2008$ & $14-16 / 10 / 2008$ & $36.85^{\circ} \mathrm{S} / 52.40^{\circ} \mathrm{W}$ & $37.80^{\circ} \mathrm{S} / 53.78^{\circ} \mathrm{W}$ & 14 \\
$\mathrm{OP} 28 / 2009$ & $02-04 / 11 / 2009$ & $37.97^{\circ} \mathrm{S} / 52.46^{\circ} \mathrm{W}$ & $44.67^{\circ} \mathrm{S} / 52.50^{\circ} \mathrm{W}$ & 15 \\
$\mathrm{OP} 31 / 2012$ & $14-16 / 10 / 2012$ & $39.01^{\circ} \mathrm{S} / 46.50^{\circ} \mathrm{W}$ & $43.01^{\circ} \mathrm{S} / 51.84^{\circ} \mathrm{W}$ & 17 \\
$\mathrm{OP} 32 / 2013$ & $13-18 / 10 / 2013$ & $33.04^{\circ} \mathrm{S} / 50.51^{\circ} \mathrm{W}$ & $46.01^{\circ} \mathrm{S} / 54.95^{\circ} \mathrm{W}$ & 16 \\
$\mathrm{OP} 33 / 2014$ & $18-21 / 10 / 2014$ & $22.89^{\circ} \mathrm{S} / 43.16^{\circ} \mathrm{W}$ & $42.61^{\circ} \mathrm{S} / 54.86^{\circ} \mathrm{W}$ & 20 \\
OP34 / 2015 & $12-15 / 10 / 2015$ & $33.04^{\circ} \mathrm{S} / 48.5^{\circ} \mathrm{W}$ & $41.21^{\circ} \mathrm{S} / 56.24^{\circ} \mathrm{W}$ & 16 \\
\hline
\end{tabular}

Total

130

\section{Dados de Reanálise}

Para identificar a influência das condições atmosféricas de grande escala sobre as concentrações de vapor d'água atmosférico em superfície e dentro da CLAM, foram utilizados dados de reanálise para os períodos dos cruzeiros. Os sistemas atmosféricos foram analisados para cada ponto de coleta de dados através do horário de reanálise mais próximo ao horário da coleta e, com base nestes dados, foram identificados os sistemas que atuaram sobre cada ponto e sua influência no comportamento do vapor d'água dentro da CLAM.

Os dados utilizados são provenientes das CFSR (Climate Forecast System Reanalysis) para o período 1999 a 2011 e sua continuação é proveniente do CFSv2 (Climate Forecast System, Versão 2) para o período 2011 a 2014, que passou a ser operacional no NCEP a partir de março de 2011. Todos os detalhes sobre as reanálises são encontrados no sítio <http://cfs.ncep.noaa.gov/cfsr/docs/> e em Saha et al. (2010, 2014).

As reanálises contam com uma resolução espacial de $0,5^{\circ} \mathrm{Lat} / \mathrm{Lon}$ e resolução temporal de seis horas, sendo um modelo totalmente acoplado, que representa a interação oceano-atmosfera-terra e gelo. O CFSv2 utiliza as mais recentes abordagens científicas e assimila observações de fontes de dados, incluindo observações de superfície, observações de balão de ar superior, observações de aeronaves e observações de satélite.

Os dados oceanográficos foram coletados pelos seguintes instrumentos: para medidas de temperatura em função da profundidade, utilizouse o XBT (Expendable Bathy Thermograph); para medidas de condutividade, temperatura e pressão, utilizou-se o CTD (Conductivity Temperature Depth). Esta medida de condutividade pode ser corrigida com a temperatura para fornecer perfil de salinidade. E para a aquisição contínua de dados de temperatura e salinidade na superfície do mar, utilizou-se o Termossalinógrafo, que é instalado no casco do navio e permite a cada 10 segundos essa aferição ao longo da trajetória realizada pelo navio, cruzando a CBM.

\section{Dados de Satélite - Sensor Modis/AQUA}

O satélite Aqua transporta, entre outros sensores, o principal deles, o sensor MODIS, desenvolvido para atender diferentes estudos 
como atmosfera, oceano e terra. O sensor tem bandas de resolução espectral e espacial selecionadas para o conhecimento de diferentes necessidades de observação e para oferecer uma cobertura global quase diária (Justice et al., 2002). A região de estudo foi separada entre lado quente e lado frio da CBM de acordo com a posição e as características, que foram selecionadas de acordo com as imagens do satélite AQUA/MODIS e com o auxílio de dados de amostragens oceanográficas coletados de instrumentos como XBTs e CTDs.

\section{Tratamento e Análise de dados}

\section{a. Estimativa do Topo da Camada Limite Atmosférica Marinha}

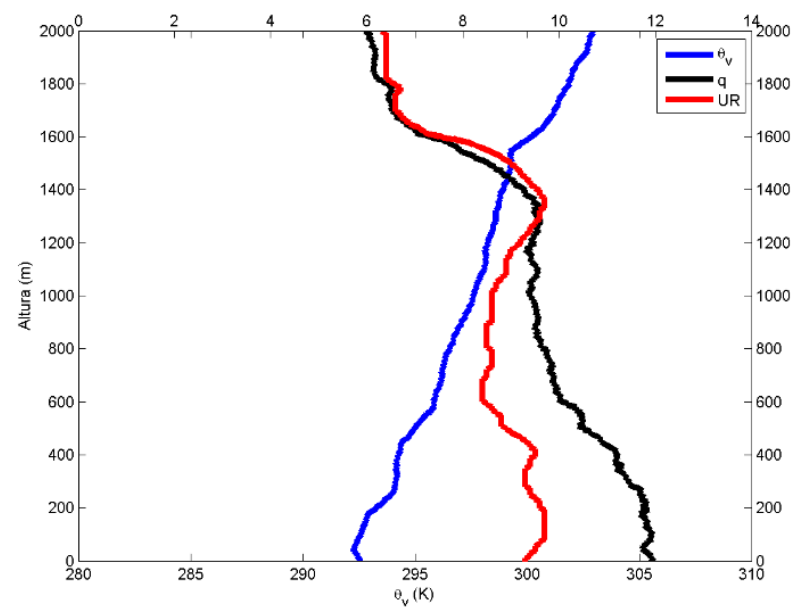

radiossondas, o topo da CLAM pode ser determinado utilizando a metodologia proposta por Stull (1988). Esse método clássico determina a altura do topo (ou espessura) da CLAM pela detecção precisa da mais pronunciada declividade dos perfis destas variáveis com respeito à altura. Em casos de dúvidas ou incertezas, e como método auxiliar, utilizou-se também o método objetivo de Sullivan et al. (1998), que foi usado com sucesso por Pezzi et al. (2009), com dados complementares aos usados nesse trabalho. A estimativa da altura do topo da CLAM foi feita para todos os perfis de radiossondas tomados in situ utilizados neste estudo.

Um exemplo de aplicação desses métodos para determinar a altura do topo da CLAM sobre a porção quente $(\mathrm{CB})$ e fria $(\mathrm{CM})$ da região deste estudo durante a realização da Operação Antártica 32 (OP32) é mostrado na Figura 2.

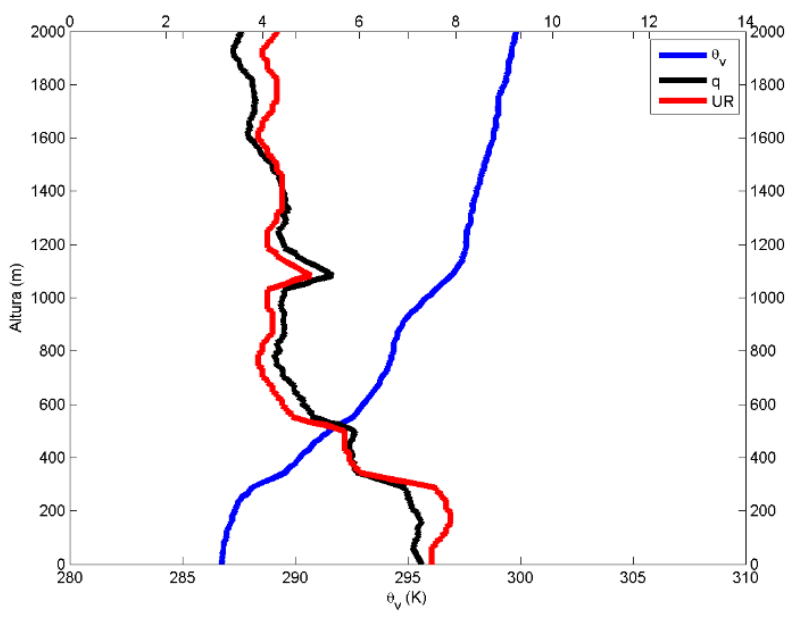

Figura 2 Perfis verticais de $\theta v(\mathrm{~K}), \mathrm{q}(\mathrm{g} / \mathrm{kg})$ e UR (x101 \%) tomados por radiossondas durante a OP31 sobre o lado quente da CBM (superior) e sobre o lado frio da CMB (inferior). A altura onde acontecem as maiores inflexões nos perfis analisados denota a altura do topo da CLAM, cerca de $1700 \mathrm{~m}$ de altura na porção quente e cerca de $400 \mathrm{~m}$ de altura na porção fria da CBM.

Através dos perfis verticais de temperatura potencial virtual $(\theta \mathrm{v})$, umidade relativa (UR) e umidade específica (q) tomados pelas

\section{b. Umidade específica}

O vapor d'água atmosférico em níveis superficiais será analisado considerando a umidade específica (q), calculada através de dados de umidade relativa provenientes das radiossondagens feitas durante os cruzeiros. Segundo Wallace e Hobbs (2006), dentro de um certo volume de ar, a quantidade de vapor d'água pode ser definida como a razão entre a massa de vapor de água (mágua) e a massa de ar seco (mar seco), denominada de razão de mistura (r), mostrado nas equações 1 e 2 . A proporção da mistura é geralmente expressa em gramas de vapor de água por quilograma de ar seco $(\mathrm{g} / \mathrm{kg})$.

$$
\mathrm{r} \text { = mágua / mar seco }
$$

A massa do vapor de água (mágua) em uma unidade de massa de ar (ar seco mais vapor de 
água) é chamada de (q) e definida como:

$$
q \frac{m_{\text {água }}}{m_{\text {água }}+m_{\text {ar seco }}}=\frac{r}{1+r}
$$

Uma vez que a magnitude de mágua é apenas uma pequena percentagem (no máximo $2 \%$ ), segue que os valores numéricos de (r) e (q) são praticamente equivalentes. Para propósitos práticos, usa-se neste estudo a umidade específica. c. Água Precipitável

O conteúdo de vapor d'água de uma coluna de ar (IWV - Integrated Water Vapor) é avaliado pela água precipitável, sendo definido como a altura de água que seria acumulada se todo vapor d'água naquela coluna fosse precipitado. Calculase IWV segundo o método proposto por Solot (1939), mais tarde adaptado por Brow e Zang (1997), mostrado na equação 3. Considerando (q) como sendo a umidade específica $\left(\mathrm{kg} \cdot \mathrm{m}^{-2}\right),(\mathrm{g})$ como a aceleração da gravidade e (pi) e (po) as pressões no topo da camada e na superfície, respectivamente, IWV é a quantidade de vapor d'água contida numa coluna vertical da atmosfera de área unitária $\left(\mathrm{kg} \cdot \mathrm{m}^{-2} ; \mathrm{mm}\right)$. Lembramos que $1 \mathrm{~kg}$ de vapor d'água sobre $1 \mathrm{~m}^{2}$ equivale a uma camada $1 \mathrm{~mm}$ e, por consequência, $1 \mathrm{~kg} \cdot \mathrm{m}^{-2}=1 \mathrm{~mm}$.

$$
(\mathrm{IWV})=\frac{1}{\mathrm{~g}} \int_{\mathrm{pi}}^{\mathrm{po}} \mathrm{qdp}
$$

Calculou-se o valor de IWV na espessura da CLAM na região desse estudo, considerando a estimativa da altura do topo da CLAM para cada perfil de radiossonda. Dessa forma, descreve-se o conteúdo vapor d'água integrado verticalmente em uma coluna de ar contida dentro da CLAM.

\section{Resultados e Discussão}

\section{Estimativa do vapor d'água em níveis superficiais e dentro da CLAM}

Tendo como referência a identificação do lado da CBM onde foi feito o lançamento da radiossonda (lado quente mais a norte, lado frio mais a sul) e dos sistemas atmosféricos que atuavam sobre cada ponto de amostragem, foi feita a análise do vapor d'água dentro da CLAM e em níveis superficiais para ambos os lados da CBM, separadamente, e para cada ponto de radiossondagem (RS).

A Figura 3 (esquerda) mostra a relação entre a temperatura da superfície do mar e água
Como ela é expressa em unidades de massa, não é afetada por mudanças na pressão ou temperatura.

A análise do vapor d'água em superfície é feita para dados a 10 metros de altura a fim de que os dados das radiossondas estejam estabilizados e descartadas as influências de turbulências e sprays gerados pelo navio e pela superfície do mar. Estes cuidados são tomados seguindo estudos anteriores, que observaram que eles podem ajudar a evitar distorções na tomada de dados in situ (Large e Pond, 1982).

precipitável dentro da CLAM, em que o comportamento do vapor d'água é investigado em relação às alterações de TSM sobre ambos os lados da CBM (Freitas et al., 2017). A média de IWV do lado quente é de $8,35 \mathrm{~mm}( \pm 4,10)$, para a Corrente das Malvinas, é de 5,71 mm $( \pm 5,00)$. A média da TSM do lado quente é de $16,98( \pm 2,57)$ e do lado frio, é de 11,30 ( $\pm 2,85)$.

Observa-se que o comportamento do vapor d'água sobre a região da $\mathrm{CBM}$ mostra que a tendência de vapor d'água atmosférico é dominada pela tendência da TSM, sendo que um aumento na TSM representa um aumento no vapor d'água atmosférico, e as maiores concentrações de vapor d'água estão sobre o lado em que a temperatura da superfície do mar é mais quente (lado mais ao norte).

Esses resultados estão em consonância com Gafinkel et al. (2013), que desenvolveram um estudo sobre as tendências de temperatura na troposfera e estratosfera, analisando suas conexões com a temperatura da superfície do mar e as implicações no vapor d'água e ozônio nos três oceanos em regiões tropicais, porém, este artigo mostra que não só a TSM domina a estrutura da concentração de vapor d'água, mas também outros fatores como os sistemas sinóticos atuantes na região de estudo, como será mostrado na sequência deste artigo.

A concentração de vapor d'água da região também é analisada associada à estabilidade estática, pois, segundo Orlanski (1986), a estabilidade estática atmosférica influencia diretamente o movimento vertical dos fluxos, podendo intensificar ou inibir os movimentos verticais. Por exemplo, uma redução da estabilidade estática nos primeiros 1000 metros da atmosfera pode influenciar na transferência de calor do oceano para a atmosfera, podendo favorecer o desenvolvimento de ciclones. 

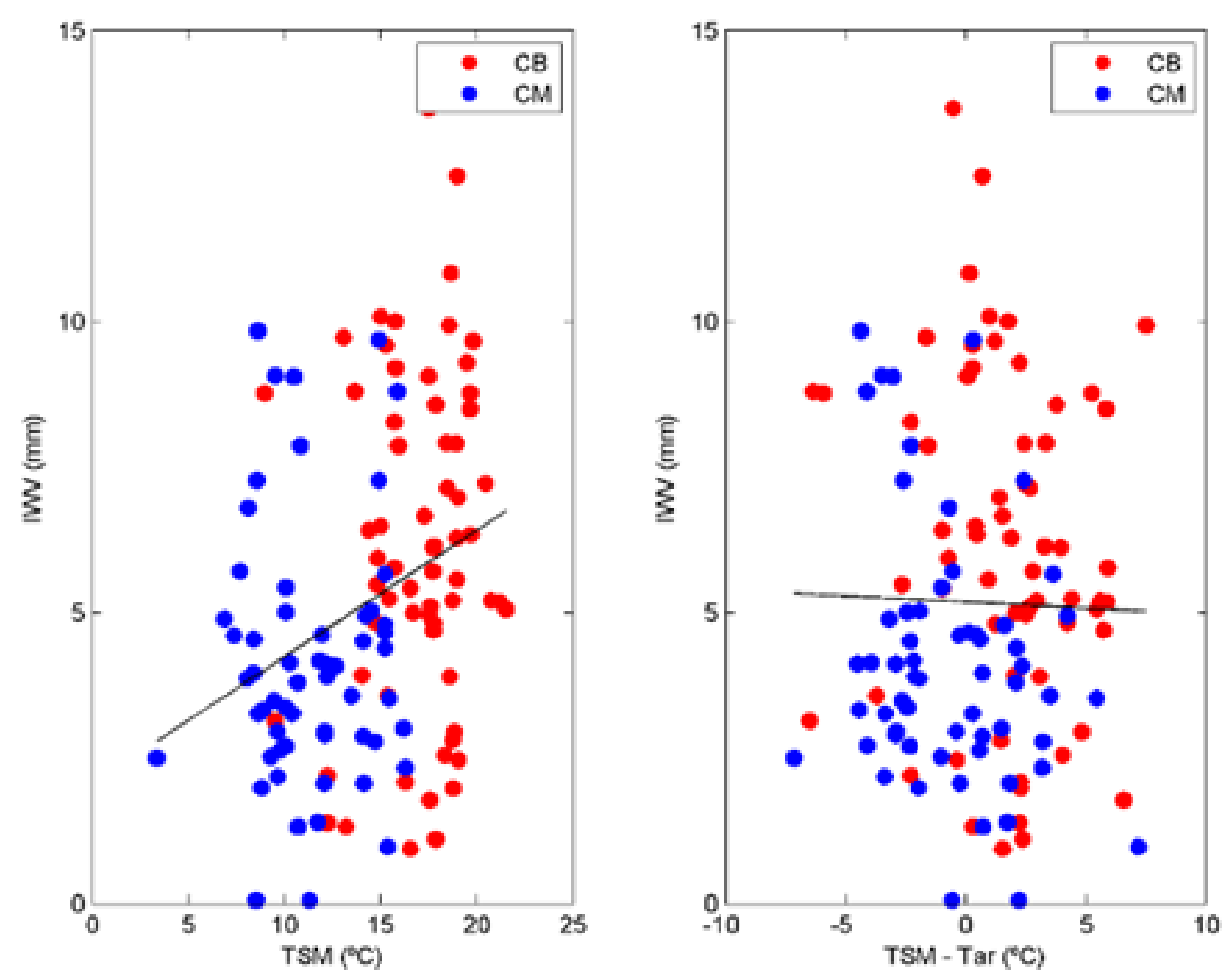

Figura 3 (Lado esquerdo) Diagrama de dispersão do domínio da média da TSM $\left({ }^{\circ} \mathrm{C}\right)$ e IWV. (Lado direito) Diagrama de dispersão entre dados de instabilidade atmosférica (TSM-Tar) e IWV (mm). Pontos sobre a CBM com preenchimento representam o lado quente e pontos sem preenchimento representam o lado frio.

Figura 3, analisa-se como o vapor d'água pode ser afetado pela turbulência presente na CLAM. Usa-se o parâmetro de estabilidade, conforme metodologia adotada por Pezzi et al. (2009), definido como TSM-Tar $\left({ }^{\circ} \mathrm{C}\right)$. Quando TSM<Tar, os valores são negativos, mostrando que a atmosfera se apresentava estável. Quando TSM>Tar, os valores são positivos, evidenciando uma atmosfera instável. Os dados plotados relacionando o parâmetro de instabilidade com o IWV têm correlação negativa, pois quanto mais estável a atmosfera se apresentar ao ser analisada no sentido de maior instabilidade, a tendência passará a ser positiva.

$\mathrm{Na}$ superfície, o vapor d'água é avaliado pela análise da umidade específica (q), evidenciando que o vapor d'água apresenta maiores concentrações sobre o lado quente da CBM, com valor médio de $8,40 \mathrm{~g} / \mathrm{kg}( \pm 1,67)$. Sobre o lado frio da CBM, a concentração de vapor d'agua apresenta seu valor médio em torno de 7,08 $\mathrm{g} / \mathrm{kg}( \pm 1.51)$.

Os perfis médios de vapor d'água na superfície para cada operação são mostrados na Figura 4 para ambos os lados da CBM, separadamente. Para testar a hipótese de que as diferenças entre os valores médios de umidade específica nas regiões quente e fria da CBM são significantes ao nível de 95\% $(\mathrm{p}<0,05)$, foi utilizado o Teste-t de Student (Wilks, 2006). O Teste-t indica que as diferenças entre os valores de q para os dois lados da frente são significativas $(\mathrm{T}=5,736 ; \mathrm{p}<0,006)$.

Os resultados mostram, por meio dos valores médios de umidade específica em superfície (q) para todas as operações analisadas, que, durante a OP31, a maior concentração estava sobre o lado frio da CBM, motivando a investigação sobre este aspecto diferenciado das demais operações. 


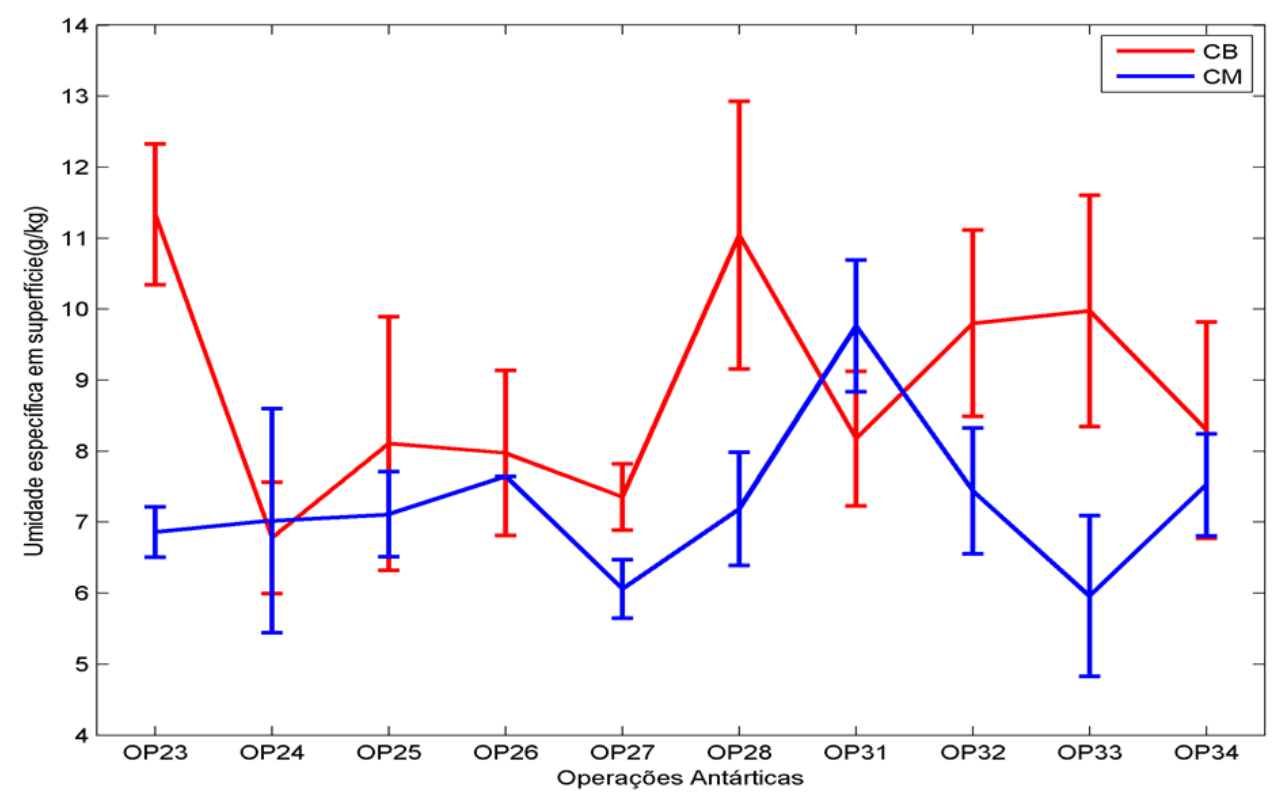

Figura 4 Valor médio dos perfis de umidade específica em superfície $(\mathrm{g} / \mathrm{kg})$ e desvio padrão $(\bar{\sigma})$ para cada Operantar sobre o lado quente e lado frio da CBM. Datas de realização: OP23 (2-3/11/2004), OP24 (28/10/2005), OP25 (27-28/10/2006), OP26 (16/10/2007, OP27 (14-16/10/2008), OP28 (02-04/11/2009), OP31(14-16/10/2012), OP32 (13-18/10/2013), OP33(18-20/10/2014) e OP34 (12-15/10/2015).

As concentrações de vapor d'água em níveis superficiais foram avaliadas também pelo cálculo do Coeficiente de Correlação de Pearson, obtendo $\mathrm{r}=0,767$ entre umidade específica em superfície e TSM. Este resultado mostra forte correlação positiva entre concentração de vapor d'água em níveis superficiais da atmosfera e temperatura da superfície do mar, concordando com estudos anteriores, que evidenciam que, em níveis superficiais, a TSM e as frentes oceanográficas modulam o vapor d'água.

\section{Estudo de caso}

Neste estudo, analisa-se a OP31, buscando responder os motivos pelos quais houve maior concentração de vapor d'água em superfície sobre o lado frio da CBM na OP31, tendo o vapor d'água em superfície se apresentado com padrões diferentes dentre os dez cruzeiros analisados.

O Teste-t de Student por grupo foi utilizado também para testar a hipótese de que as diferenças entre os valores de (q) nas regiões quente e fria da CBM durante a OP31 são significativas ao nível de $95 \%(\mathrm{p}<0,05)$. O Teste$t$ mostrou que as diferenças entre os valores de $q$ para os dois lados da frente durante a OP31 são significativas $(\mathrm{T}=-2,87 ; \mathrm{p}=0,012)$.

As condições atmosféricas durante o cruzeiro feito pelo INTERCONF na OP31 são analisadas segundo os campos de pressão ao nível médio do mar (hPa), vento médio em $1000 \mathrm{hPa}$ $\left(\mathrm{m} . \mathrm{s}^{-1}\right)$, umidade específica $(\mathrm{g} / \mathrm{kg})$ e linhas de corrente em $850 \mathrm{hPa}$. Foram também utilizadas para análise imagens de satélite. Como mencionado anteriormente, para definir o lado da CBM em que os sistemas atuavam, foram usadas imagens do satélite AQUA/MODIS e dados oceanográficos coletados in situ e classificadas conforme sua posição na região de estudo: lado quente (mais ao norte) e lado frio (mais ao sul) da CBM.

Durante a realização da OP31, foram feitos dezesseis lançamentos de radiossondas entre os dias 14 e 16 de outubro de 2012: 10 radiossondas foram lançadas sobre o lado quente da CBM, posteriormente, 06 radiossondas, sobre o lado frio da CBM. Neste período, a região da CBM esteve sob a atuação de dois sistemas de alta pressão, obtendo uma amostragem sob uma atmosfera mais seca durante o cruzeiro, evidenciada nos campos de umidade em superfície mostrados na Figura 5 (lado direito). 

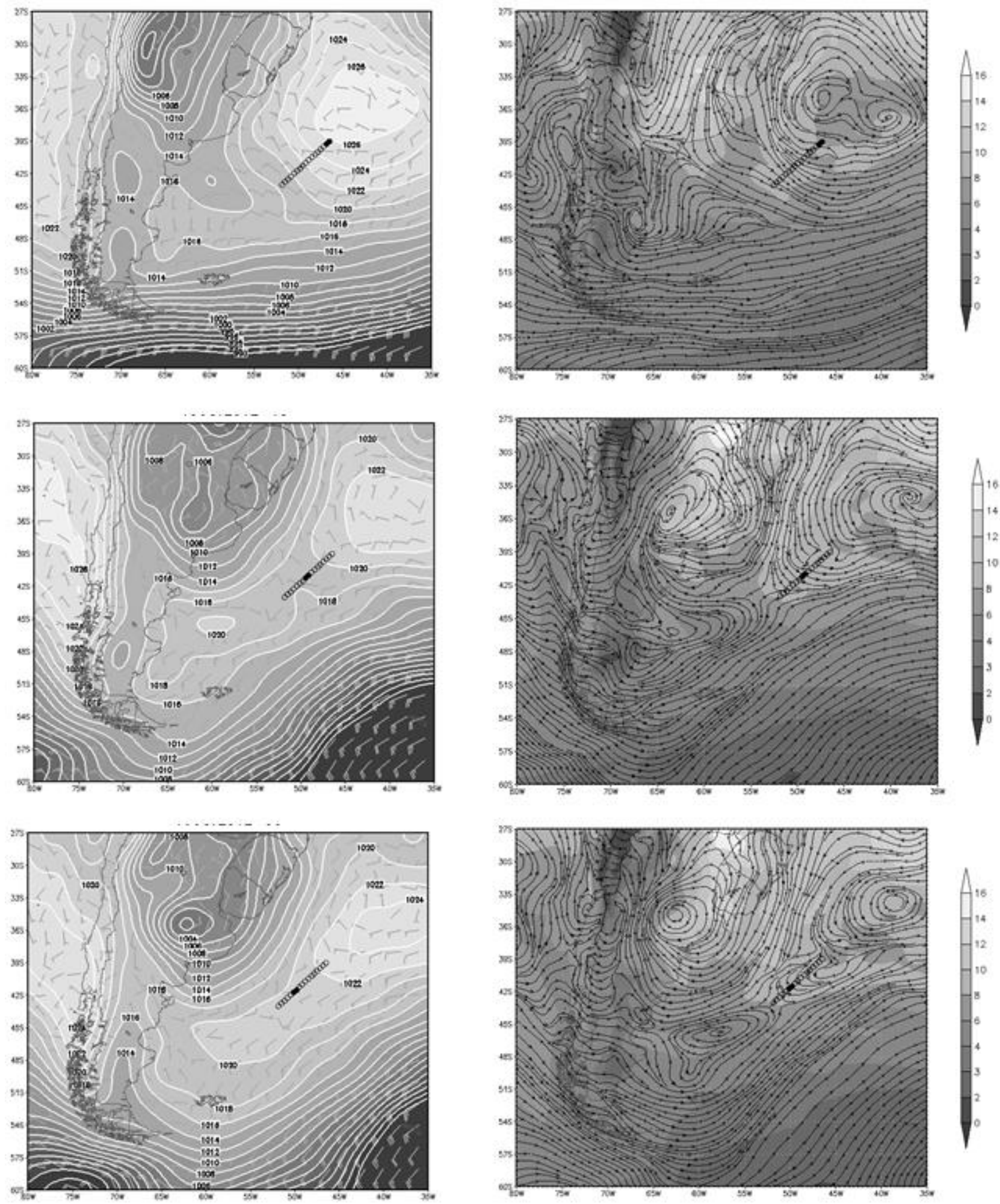

Figura 5 Condições atmosféricas durante a OP31 a partir de dados de reanálise CFSv2. As figuras à esquerda são de pressão atmosférica ao nível médio do mar $(\mathrm{hPa})$ e vento médio $1000 \mathrm{hPa}\left(\mathrm{m} . \mathrm{s}^{-1}\right)$ e, na direita, umidade específica a 2 metros $(\mathrm{g} / \mathrm{kg}$ ) e linhas de corrente em $850 \mathrm{hPa}$. As estações de lançamentos de radiossondas estão marcadas pelos círculos pretos.

Os primeiros pontos de coleta de dados de RS (RS01 a RS06) estavam sobre a CB sob a influência de um anticiclone transiente que, posteriormente, se fundiu com a Alta Subtropical do Atlântico Sul (ASAS), como se pode observar através dos campos de pressão ao nível médio do 
mar e vento médio $1000 \mathrm{hPa}$, pontos sob atuação de ventos de Norte $(\mathrm{N})$ de forte intensidade. No segundo dia de coleta de dados, houve aproximação de um outro sistema anticiclônico transiente mostrado na RS11, marcando o encontro destes dois sistemas pelo enfraquecimento do vento, logo depois, o sistema transiente incorporase a ASAS, tornando-se um só sistema de alta pressão.

Os pontos amostrados durante a OP31 apresentaram-se em maior parte sob condições de advecção quente, Figura 6, especialmente quando os sistemas de alta pressão se fundem, passando a ASAS a ter influência na região especialmente sob condições de advecção quente sobre o lado frio da CBM. Os campos de umidade em superfície mostrados anteriormente apresentam características semelhantes com os pontos amostrados.

Segundo Mächel et al. (1998), uma análise feita com mais de cem anos de dados para descrever a variação espacial da ASAS ao longo do ano mostrou que, entre os meses de maio e outubro, a ASAS varia bastante em relação à sua intensidade e abrangência espacial. No mês de outubro, mês de realização de maior parte dos cruzeiros analisados neste estudo, a ASAS normalmente se encontra em torno de $2^{\circ} \mathrm{W}$. Degola (2013) mostrou que a posição da ASAS no mês de outubro adentra o continente sul-americano, tendo maior influência no clima das regiões Sudeste e Nordeste do Brasil e, consequentemente, influencia a região da CBM, como demonstrado nos resultados do presente estudo.

A Figura 7 mostra a concentração do vapor d'água atmosférico integrado verticalmente até o topo da CLAM (IWV) para cada ponto de coleta de dados sobre a CBM durante a OP31, sob presença de sistemas sinóticos distintos. A linha preta tracejada representa o parâmetro de instabilidade e a linha vermelha sólida representa IWV. Em estudo recente, De Freitas et al. (2017), por meio de dados observacionais, mostraram que a altura média da CLAM no lado quente (CB) é de $951,25 \mathrm{~m}$ e ( $\bar{\sigma} \bar{\sigma})$ 442,63 , enquanto no lado frio (CM), a altura média é de 800,32 e ( $(\bar{\sigma}) 422,00$.
A análise dos campos de IWV durante a OP31 mostrou que, em sua maior parte, eles estão sob atuação de sistemas anticiclônicos, evidenciando que a região está sob atuação de sistema de alta pressão com movimento de subsidência de ar onde a região está mais seca, estando as maiores concentrações sobre os pontos coletados sobre o lado mais quente (RS1 a RS6). Observa-se também uma relevante situação em que a RS11, lançada sob a latitude $41.5^{\circ} \mathrm{S}$, mostra uma rápida diminuição da concentração de vapor d'água mostrada verticalmente ainda sobre a $C B$, estando esse ponto sobre uma área de encontro dos sistemas de circulação, que acontece pela aproximação do segundo sistema transiente de alta pressão, sendo que, neste ponto, a atmosfera passa a apresentar desintensificação do vento, influenciando diretamente na IWV. A análise da OP31 juntamente com todos os outros cruzeiros analisados, não mostrados neste estudo, comprovam que, quando há uma região de máximo gradiente termal, mostrado pelo encontro das duas frentes, normalmente há um decréscimo rápido de vapor d'água, caracterizando a região de encontro dos lados quente e frio da CBM. Este ponto de amostragem pode ser notado na Figura 7, aproximadamente em $40^{\circ} \mathrm{S}$.

A análise tendo como referência os dados observados na região mostra que a água precipitável não é definida somente em relação à temperatura da superfície do mar na região da CBM, mas também está altamente relacionada aos sistemas de grande escala atuantes na região, que estão altamente relacionados à advecção de temperatura sobre a superfície oceânica. Estudos anteriores como os de Stephens (1989) correlacionam dados da TSM com dados de água precipitável, com base na equação de ClausiusClayperon, e afirmam que o teor de vapor d'água na atmosfera acima dos oceanos pode ser prescrito pela temperatura da superfície do mar, com desvio padrão de $0,36 \mathrm{~g} / \mathrm{cm}^{2}$. Mostram estes estudos que, dentro da CLAM, devem ser considerados efeitos oceânicos e atmosféricos para considerar IWV na região. 

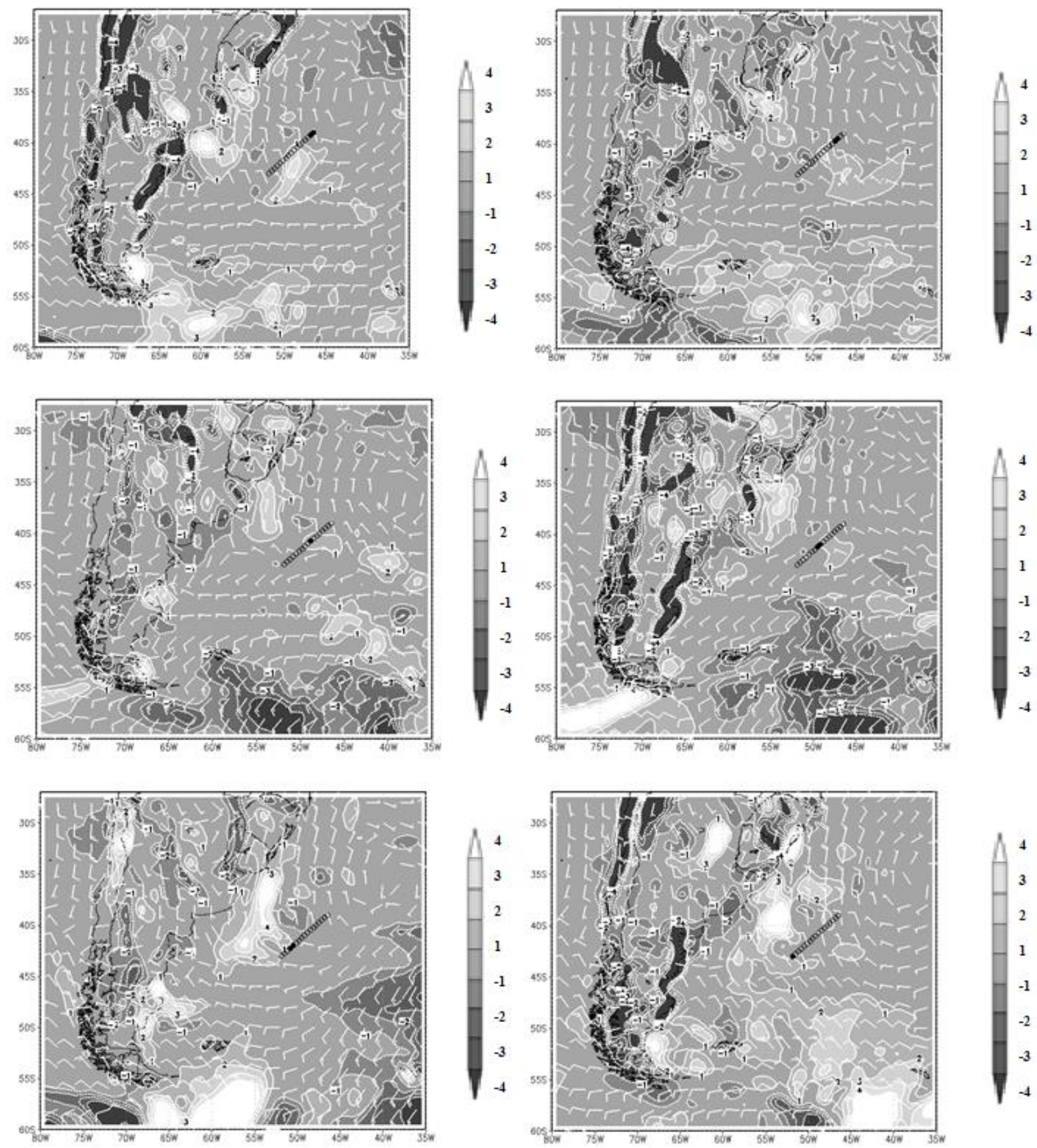

Figura 6 Advecção térmica $\left({ }^{\circ} \mathrm{C} / 6\right.$ horas) e vento $\left(\mathrm{m} . \mathrm{s}^{-1}\right)$, em $1000 \mathrm{hPa}$ durante a OP31, considerando dados de reanálise. As estações de lançamentos de radiossondas estão marcadas pelos círculos pretos.

Na Figura 7 também se analisa o parâmetro de estabilidade do ar na concentração de vapor d'água integrado na região da CBM para OP31. Pode-se observar que, na maior parte do deslocamento do navio, a temperatura da superfície do mar foi maior que a temperatura do ar, indicando condição de instabilidade. Pode-se observar que onde existem valores negativos indicando que a atmosfera se apresenta estável sobre a CB, o vapor d'água atmosférico alcança suas menores concentrações. $\mathrm{O}$ mesmo fenômeno pode ser observado sobre a CM, em que a estabilidade estática se apresenta em quase toda trajetória com valores positivos, mostrando a instabilidade da atmosfera sobre a CM, mas a concentração de vapor d'água se apresentando bem menor. Para fundamentar tal afirmação, são analisados os dados referentes a OP31 na Figura 8, que mostra o gráfico de dispersão das observações para a OP31. A Figura 8 permite analisar a relação entre a umidade específica em níveis superficiais e TSM, Figura 8a, e a instabilidade estática dada pela 
TSM-Tar (Figura 8b). Percebe-se que a umidade específica nas OP31 em níveis superficiais apresentou comportamento esperado, aumentando conforme aumenta a TSM, e tem seus pontos de coleta distribuídos entre regiões estáveis e instáveis, e quanto maior a instabilidade da região, maior a concentração de vapor d'água superficial.

Na Figura 8 também referente à OP31, são analisadas as relações entre IWV contido na CLAM e TSM, Figura 8c, e entre IWV e instabilidade estática, dada pela TSM-Tar (Figura 8d). Nota-se claramente que a concentração de vapor d'água dentro da CLAM, dada pela água precipitável, aumenta com o aumento da TSM. Por outro lado, essa relação não é clara em relação ao parâmetro de estabilidade. Nota-se que na OP31 essa relação não é direta, provavelmente pela presença de sistemas atmosféricos anticiclônicos durante todo o cruzeiro.

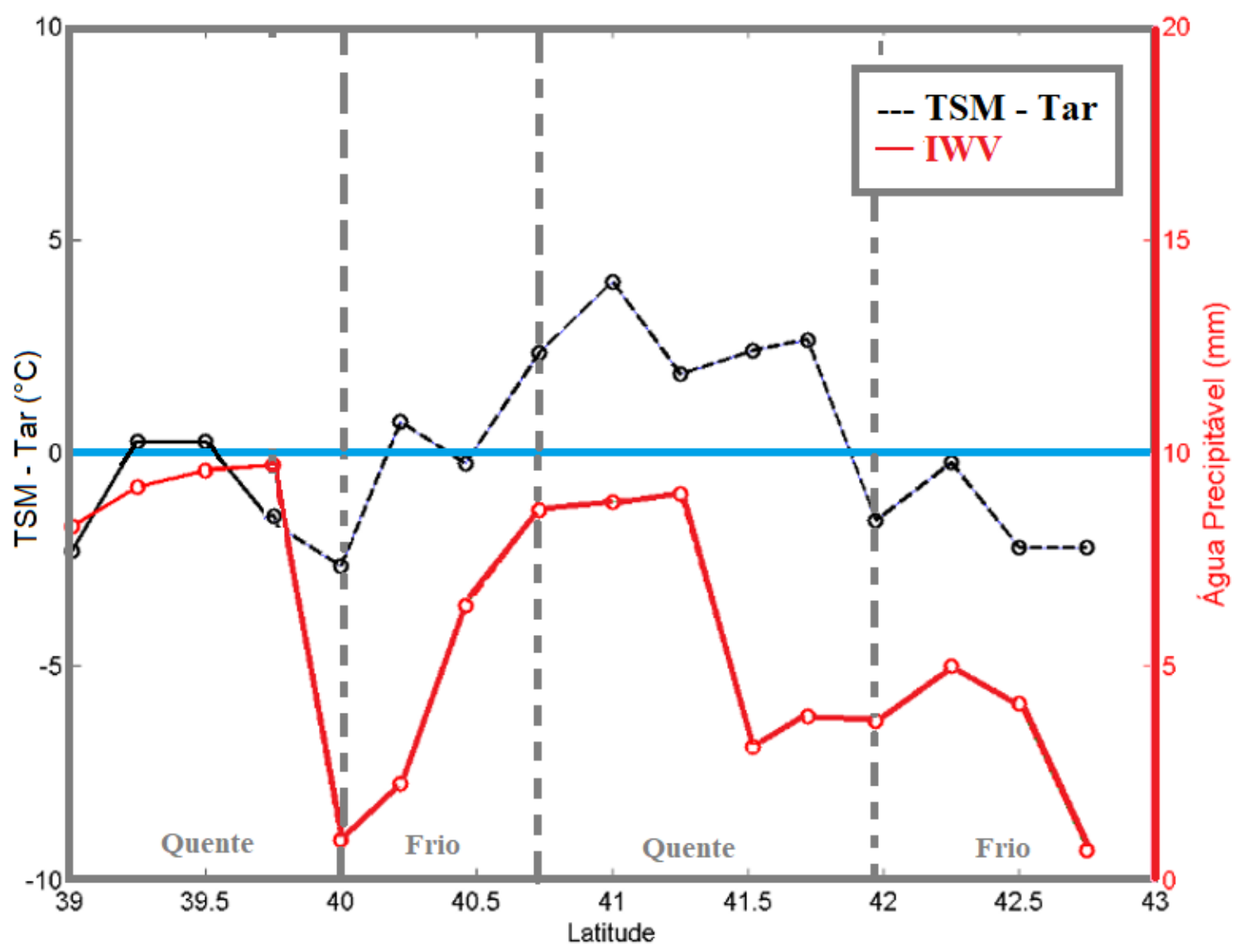

Figura 7 Variação do parâmetro de estabilidade TSM-Tar (linha preta) e conteúdo de água precipitável (linha vermelha) dentro da CLAM ao longo das OP31, considerando dados coletados in situ. Linha vertical pontilhada cinza indica separação de água mais quente e mais fria na CBM, linha azul representa a TSM em $0^{\circ} \mathrm{C}$. 

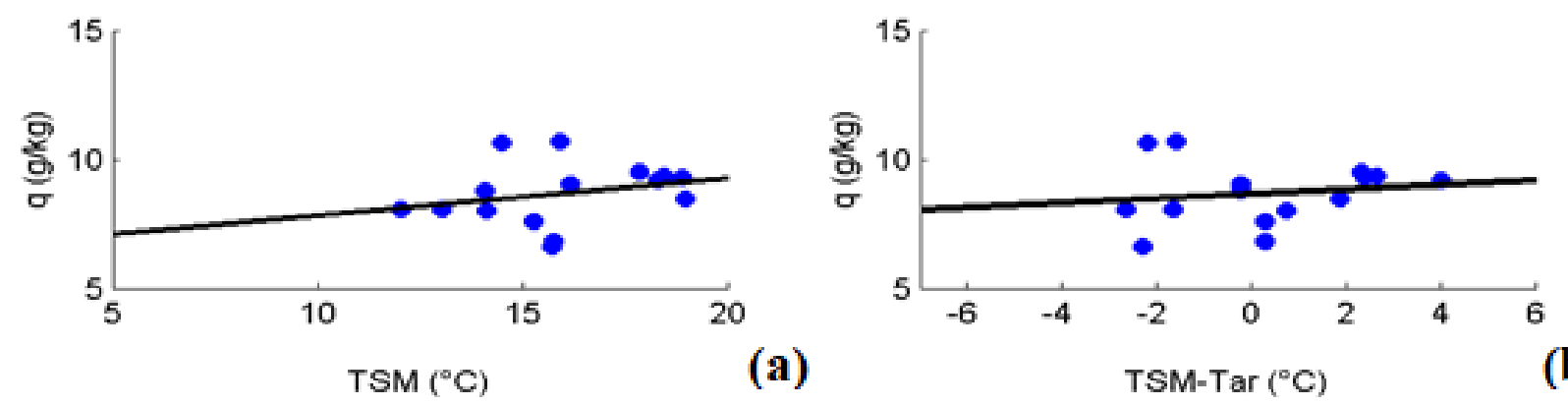

(b)
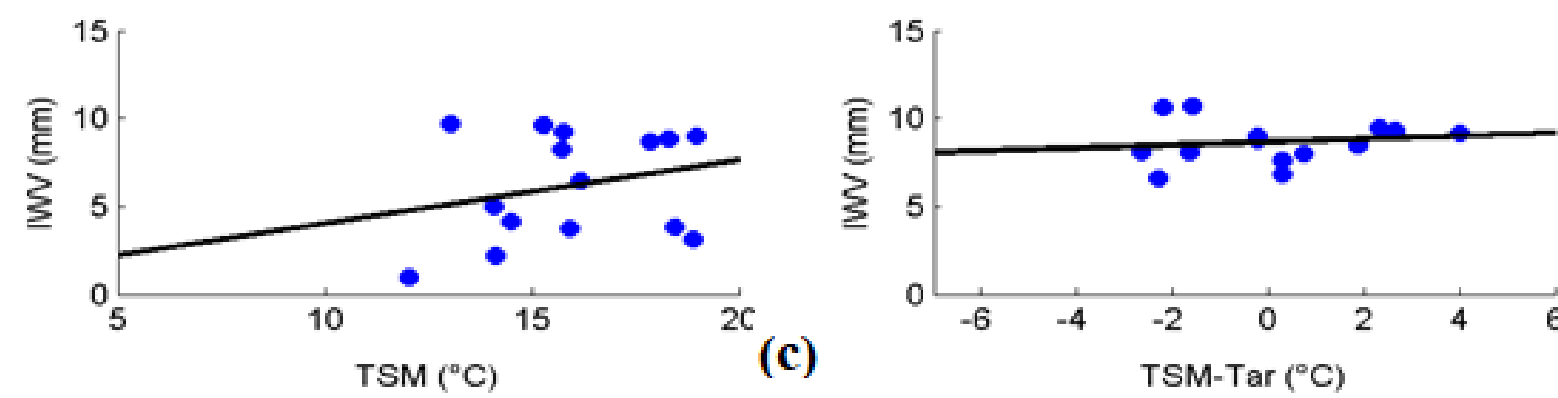

(d)

Figura 8 Variação da umidade especifica em superfície durante a OP31 (a) em relação à TSM (b) em relação à instabilidade estática. Variação do vapor d'água verticalmente integrado na CLAM durante a OP31 (c) em relação à TSM e (d) em relação à instabilidade estática.

\section{Conclusões}

Tendo em vista a carência de dados observados no Hemisfério Sul, são de grande relevância medições feitas durante os dez anos de coleta de dados pelo Programa INTERCONF, proporcionando uma análise do sistema acoplado oceano-atmosfera na região da CBM através de amostragens verticais da atmosfera por meio de radiossondas e também das características oceânicas por meio de equipamentos apropriados.

Este artigo teve por finalidade analisar o vapor d'água dentro da CLAM na região da CBM, através de dados coletados in situ, tendo os resultados demonstrado, através da análise das médias e desvio padrão $(\bar{\sigma})$ das 130 estações de coletas de dados atmosféricos e oceanográficos, que a TSM média do lado quente é de $16,98{ }^{\circ} \mathrm{C}( \pm$ $2,57)$ e para o lado frio, é de $11,30{ }^{\circ} \mathrm{C}( \pm 2,85)$, enquanto a umidade específica apresentou valores médios em superfície de $8,40 \mathrm{~g} \cdot \mathrm{kg}^{-1}( \pm 1,67)$ e para o lado frio, de 7,08 g.kg-1 ( $\pm 1,51)$. Considerando a camada limite atmosférica marinha, a análise dos dados mostrou que o conteúdo de vapor d'água integrado dentro da CLAM, representado pelo IWV, também é influenciado pela TSM e pela instabilidade, especialmente sobre o lado quente da CBM. A média de IWV do lado quente foi de 8,35 $\mathrm{mm}( \pm 4,10)$, e para o lado frio da CBM foi de 5,71 $\mathrm{mm}( \pm 5,00)$, os resultados corroboram com Freitas et al., (2017).

A região da CBM pode ser influenciada tanto por sistemas transientes como por sistemas semipermanentes (ASAS). Neste estudo, foi observada influência dos sistemas transientes na região. Os resultados indicam que o deslocamento dos sistemas transientes tem influência sobre a região, podendo aumentar/diminuir a advecção de temperatura/umidade, influenciando diretamente no vapor d'água sobre a região. Por outro lado, a predominância do vento de Oeste sobre o lado quente e do vento Norte sobre o lado frio da CBM, associada ao deslocamento para leste de sistemas sinóticos transientes, induz advecção de temperatura e umidade sobre a região, influenciando diretamente nas características das variáveis dentro da CLAM, por processos induzidos pelo oceano. Estes processos são influenciados pela velocidade do vento, tais como processos turbulentos que transportam vapor d'água na região, influenciando nas concentrações de vapor d'água na região.

Estes resultados juntamente com as análises mostradas indicam que a TSM age como força local, modulando o vapor d'água atmosférico, mantendo maior concentração em níveis superficiais e passando a ser modulada nos níveis superiores pelos sistemas de grande escala que atuam na região. 
A região da $\mathrm{CBM}$ pode, através de sistemas transientes, deslocar vapor d'água e influenciar diretamente na região Sul e Sudeste do Brasil. Sugere-se para estudos futuros que sejam consideradas as diferenças nas concentrações de vapor d'água sobre a região na melhoria de modelos numéricos de previsão de tempo e clima, considerando temporal e espacialmente as análises mostradas neste estudo.

\section{Agradecimentos}

Os autores agradecem ao $\mathrm{CNPq}$ pela bolsa de estudo ao primeiro autor e pelo apoio a R.B. S no Programa de Produtividade em Pesquisa (308646/2013-4). Esse trabalho é uma contribuição aos projetos do Instituto Nacional de Ciência e Tecnologia da Criosfera (CNPq/PROANTAR 704222/2009) e Estudos Avançados em Oceanografia de Médias e Altas Latitudes (CAPES 23038.004304/2014-28).

\section{Referências}

Anderson, Theodore Wilbur et al. 1958. An introduction to multivariate statistical analysis. New York: Wiley.

Brown, Randy G.; ZHANG, Chidong. 1997. Variability of midtropospheric moisture and its effect on cloud-top height distribution during TOGA COARE. Journal of the atmospheric sciences 54, 2760-2774.

Cardoso Denis Harley; DOURADO, Marcelo. 2013. Modelagem unidimensional da camada limite oceânica durante a passagem de uma frente fria na região da confluência BrasilMalvinas. Revista Brasileira de Meteorologia 29, $367-378$.

De Camargo, Ricardo et al. 2013. Modulation mechanisms of marine atmospheric boundary layer at the Brazil-Malvinas Confluence region. Journal of Geophysical Research: Atmospheres 118, 6266-6280.

Freitas, Rose Ane Pereira et al. 2017. Ánalise do conteúdo de vapor d'água na camada limite atmosférica marinha sobre a região da Confluência Brasil-Malvinas entre os anos de 2004 e 2015. Anuário do Instituto de Geociências 40, 94-101.

Garfinkel, C. I. et al. 2013. Temperature trends in the tropical upper troposphere and lower stratosphere: Connections with sea surface temperatures and implications for water vapor and ozone. Journal of Geophysical Research: Atmospheres 118, 9658-9672.

Justice, C.O.; et al. 2002. The MODIS fire products. Remote Sensing of Environment 83,
244-262.

Large, W. G.; Pond, S. 1982. Sensible and latent heat flux measurements over the ocean. Journal of physical Oceanography 12, 464-482.

Mächel, H.; Kapala, A.; Flohn, H. 1998. Behaviour of the centres of action above the Atlantic since 1881. Part I: Characteristics of seasonal and interannual variability. International Journal of Climatology 18, 1-22.

Nygård, T., Valkonen, T., And Vihma, T. 2013. Antarctic Low Tropospheric Humidity Inversions $10 \mathrm{yr}$ Climatology, Journal of Climate 26, 5205-5219.

Orlanski, Isidoro. 1986. Localized baroclinicity- A source for meso-alpha cyclones. Journal of the atmospheric sciences 43, 2857-2885.

Pezzi. L.P.; Souza. R.B.; Acevedo. O. 2009. Multiyear measurements of the oceanic and atmospheric boundary layers at the BrazilMalvinas confluence region. Journal of Geophysical Research1 14: 1-19.

Pezzi, Luciano Ponzi et al. 2005. Oceanatmosphere in situ observations at the BrazilMalvinas Confluence region. Geophysical Research Letters DOI:10.1029/2005GL023866

Pezzi, Luciano Ponzi et al. 2016. Uma Revisão dos Processos de Interação Oceano-Atmosfera em Regiões de Intenso Gradiente Termal do Oceano Atlântico Sul Baseada em Dados Observacionais. Revista Brasileira de Meteorologia 31, 428-453.

Saha, Suranjana et al. 2010. The NCEP climate forecast system reanalysis. Bulletin of the American Meteorological Society 91, 10151058.

Saha, Suranjana et al. 2014. The NCEP climate forecast system version 2. Journal of Climate 27, 2185-2208.

Solot, Samuel B. 1939. Computation of depth of precipitable water in a column of air. Monthly Weather Review 67, 58.

Stephens, Graeme L. 1990. On the relationship between water vapor over the oceans and sea surface temperature. Journal of Climate. 3, 634645.

Stull. R.B. 1988. An Introduction to Boundary Layer Meteorology. Dordrecht. The Netherlands: Kluwer Academic Publishers. 666 p.

Sullivan, Peter P. et al. 1998. Structure of the entrainment zone capping the convective atmospheric boundary layer. Journal of the atmospheric sciences 55, 3042-3064. 
Wallace, J.M.; Mitchell, T.P.; Deser, C. J. 1989. The influence of sea surface temperature on surface wind in the eastern equatorial Pacific: Weekly to monthly variability, Journal of Climate 2: 1492 - 1499.

Wilks, D.S.; 1995. Statistical methods in the atmospheric sciences. San Diego. International Geophysics Series. Second Edition. 139p.
Xie, Shang-Ping. 2004. Satellite observations of cool ocean-atmosphere interaction. Bulletin of the American Meteorological Society 85, 195208. 